\title{
BMJ Open Qualitative study on mental health and well-being of Syrian refugees and their coping mechanisms towards integration in the UK
}

\author{
Priyamvada Paudyal (D), Mais Tattan, Maxwell J F Cooper
}

To cite: Paudyal $P$, Tattan $M$, Cooper MJF. Qualitative study on mental health and well-being of Syrian refugees and their coping mechanisms towards integration in the UK. BMJ Open 2021;11:e046065. doi:10.1136/ bmjopen-2020-046065

- Prepublication history for this paper is available online. To view these files, please visit the journal online (http://dx.doi. org/10.1136/bmjopen-2020046065).

Received 21 October 2020 Accepted 28 July 2021

\section{ABSTRACT}

Objective This study aimed to explore the mental well-being of Syrian refugees and identify their coping mechanisms and pathways towards integration into new communities.

Design Qualitative study using in-depth semi-structured interviews.

Setting and participants Adult Syrian refugees ( $>18$ years old) currently residing in South East of England. Results 12 participants ( 3 women and 9 men) took part in the study, all were born in Syria and the majority $(n=9)$ were over 45 years of age. Our findings show that Syrian refugees face constant challenges as they try to integrate into a new society. Loss of and separation from loved ones as well as the nostalgia for the homeland were often cited as a source of psychological distress that created an overwhelming sense of sadness. Participants reported that they struggled for connectedness due to cultural difference and the problematic nature of rapidly formed migrant communities in their new setting. They believed in 'being their own doctor' and turning to faith, ritual and nature for healing and comfort. Taboo and stigma around mental health and language barriers were cited as barriers to accessing mental healthcare services.

Conclusion Past experiences and present challenges frame Syrian refugees' sense of well-being, impact use of healthcare and risk future mental health problems. It is hoped that this study will act as a catalyst for further research on this vulnerable group to promote integration, community support and culturally sensitive mental health services.

\section{INTRODUCTION}

The mass movement of Syrian refugee is recognised as the biggest forced displacement predicament the world has seen since the second world war ${ }^{1}$ and was declared by the United Nation High Commissioner for Refugees as the biggest humanitarian crisis of our present time. ${ }^{2}$ Since 2011, following the escalating conflict and violence in Syria, millions have been forced to flee their homes in search of safety and protection. The most recent estimates suggest that about 5.6 million Syrians have registered as refugees
Strengths and limitations of this study

To date, this is the first qualitative study conducted among Syrian refugees in the UK.

- The interviews were conducted entirely in the language and dialect of the participating Syrian refugees.

- This study is limited by its small sample size $(n=12)$. However, the study gathered rich data and identified themes align with the findings of previous research on refugees of different backgrounds.

in neighbouring countries, and an additional 6.6million Syrians are internally displaced within the borders of Syria. ${ }^{3}$

Refugees are at risk of multiple traumatic experiences that impact their mental and physical health. These traumatic factors originate from their home country, during their displacement journey or as part of the asylum process. Preimmigration factors such as physical injury, near-death experiences, witnessing murder, bereavement (eg, the traumatic loss of loved ones), imprisonment and torture are often associated with civil conflict. ${ }^{4}$ Others encounter additional traumatic events during their journey to exile and as part of the settlement process. Evidence suggests that postimmigration stressors such as ethnic discrimination, harsh socioeconomical living conditions, institutional accommodation, limited language skills and poor social support are detrimental to refugees' mental health, and the impact may persist for years or possibly decades after resettlement to a new environment. ${ }^{5-12}$ For such reasons, Syrian refugees have been found to live with hopelessness, fear and constant worries. ${ }^{13}$

The majority of those who seek asylum in the UK come from countries that are in the midst of conflict. ${ }^{14}$ Previous research on refugees in the UK have concluded that one in six refugees develops severe health problem that 
could permanently affect their quality of life. ${ }^{15}$ Further research have found that asylum seekers face worse outcomes than the UK population on almost all measures of health and well-being. ${ }^{16}$ Symptoms of anxiety, depression, post-traumatic stress disorder (PTSD) and agoraphobia have been reported among this population, ${ }^{17}$ and it has been estimated that up to two-thirds of refugees in the UK experience anxiety or depression. ${ }^{18}$ In 2014, the UK launched its Syrian vulnerable person resettlement (VPR) programme aiming to resettle 20000 Syrian refugees across the country by the year $2020 .{ }^{19}$ The crisis has been escalated recently with powerful images in the news and social media covering stories of desperate and vulnerable people trying to cross the English Channel to reach the UK. ${ }^{20}$ In this context, there is a need for further understanding of the impact of the current refugee crisis on Syrians, particularly after resettlement. Most importantly, there is a need to explore the coping mechanisms and resilience pathways of Syrian refugees to promote their well-being, integration and transition into active members of society.

Numerous studies have reported on the prevalence of several mental health disorders among Syrian refugees. ${ }^{21-23}$ Depression, anxiety and PTSD were found to be the most prevalent disorders among displaced individuals. ${ }^{24}$ However, the existing literature is mostly comprised of quantitative studies and there is a clear need for undertaking a comprehensive qualitative research. This study aimed to explore mental well-being of Syrian refugees, barriers to seeking mental healthcare services, coping mechanisms and pathways towards integration in new communities.

\section{METHODOLOGY}

This qualitative study was conducted in South East England using semi-structured interviews. Qualitative methods were selected to allow in-depth understanding of practical issues through detailed exploration of individual's experiences, motives and opinions. ${ }^{25}$ Participants were recruited via the contacts of language centres, community organisations and charities using purposive and snowballing sampling approach. This method was chosen as purposive sampling involves identifying and selecting individuals or groups that are knowledgeable or have experienced the studied phenomenon. Adult Syrian refugees ( $>18$ years of age), currently living in South East England, and speaking Arabic or English were eligible for inclusion. For ethical reasons, details of participants' previous mental health diagnoses were not collected.

Participants were approached through the gatekeepers of the respective organisations, and a snowballing technique was used to approach further possible participants. They were provided with participant information leaflets (PIL) in the Arabic and English languages according to participants' preferences, which provided detailed information about the study. Thirty potential participants were invited to participate. Of these, 13 agreed to participate

\section{Box 1 Topic guide used for the interview process}

\section{Introduction.}

Beliefs about mental health and well-being.

- Personal definition of mental health.

- Explore why some people have good mental health and others do not.

- Ways to help oneself to have good mental health.

Community support and coping mechanism.

- Community in the UK and newly formed relationships.

- Emotional or physical challenges faced.

- Coping mechanisms to overcome the challenges.

Access to mental healthcare services.

- Understanding of mental health facilities in the UK.

Barriers and facilitators in accessing mental health carehealthcare.

and one participant withdrew before the start of the interviews. Altogether, 12 participants comprised the sample for this study.

Due to the vulnerable nature of the topic and the predicament of participants, no incentive was offered while inviting potential participants; participation was entirely voluntary and the right to withdraw was available at any point. Informed consents were obtained from participants after full explanation and reiteration of the information provided in the PIL.

A semi-structured interview topic guide (box 1) was constructed to explore participants' understanding of mental health problems and beliefs about mental health and well-being, community support, coping mechanisms and access to healthcare services. Interviews were conducted between April and June 2019 in a location of the participant's choice. Six interviews were conducted in private spaces within community organisations, and the other six were conducted in open public spaces chosen by participants, such as public parks. All interviews were conducted in Arabic, transcribed verbatim and the scripts were then translated into English.

Data were analysed using thematic content analysis following Burnard's method ${ }^{26}$. This involves a systematic 14-part method of step-by-step process of open coding and categorisation. The approach is used to evaluate and record the themes and issues highlighted in texts produced by the participants and categorised appropriately. The coding was done iteratively; random samples of the identified themes were discussed with the research team until agreement was reached for the key themes.

The researcher who collected the data (MT) is bilingual and shares the nationality and the language of the studied refugee participants (except the refugee status). This requires a reflection on the insider-outsider qualitative researcher approach. Being an insider researcher has advantages, they are trusted and accepted by the participants and have greater understanding about the phenomena being studied. However, it can be argued that this could lead to a loss of objectivity and bias. ${ }^{27}$ Hence, 
Table 1 Demographic characteristics of participants

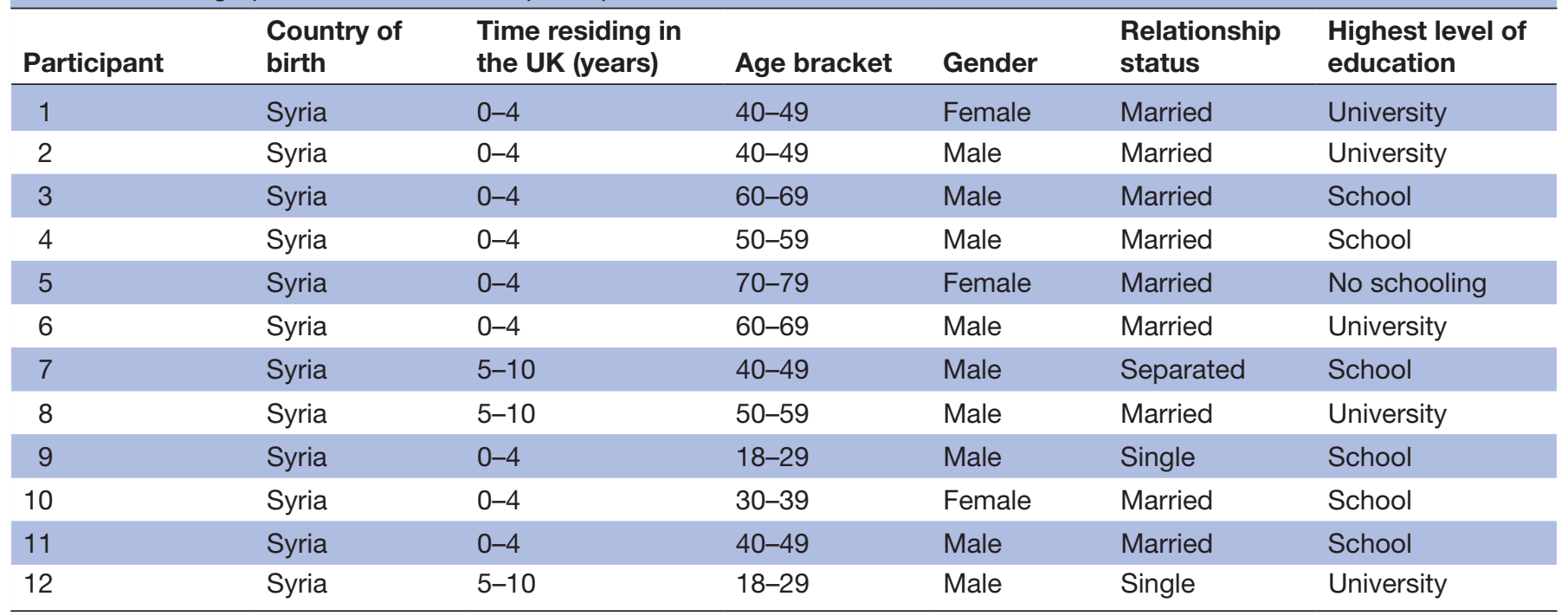

prior to the data collection, the researcher adopted the philosophy of Asselin, which recommends the insider researcher to approach research while assuming no previous knowledge of the studied phenomenon. ${ }^{28}$ The researcher was also trained to conduct the interviews in a neutral manner using open questions and encouraging the participants to express ideas and thoughts.

\section{Patient and public involvement}

Patients or the public were not involved in the design, or conduct, or reporting or dissemination plans of our research.

\section{RESULTS}

The study's sample consisted of 12 refugee participants, 3 women and 9 men (table 1 ). All participants were born in Syria and nine had moved to the UK less than 5 years ago. Three quarter of the participants $(n=9)$ were over 45 years of age, nine were married with children, one separated and two single. Five participants were university graduates, six had school-level education and one was illiterate. Average time for residing in the UK was 3.8 years, range (3 months-10 years) (table 1$)$.

\section{Identified themes}

Four major key themes were identified from the data analysis; loss and separation, struggling for connectedness in a culturally divided world, health beliefs and practices and barriers to healthcare access. Several subthemes emerged under each key theme and are elaborated on using quotes. Listing of key themes and subthemes is found in figure 1 .

\section{Loss and separation}

Participants often perceived that loss of and separation from their family members were causing or aggravating their current psychological distress. These feelings were not limited to the loss of loved ones but also the loss of material possessions, and connections to their home country in general:

Sometimes when I remember my brother who passed away... makes me feel upset... like we were six people in the family... me, my mum, my dad, and my three siblings, a brother and two sisters... suddenly there was only me, my mum and my dad (participant 9, man)

One participant described the meaning of home to a Syrian family and the consequences of its loss:

When you build a home it's like a celebration, it means you have established a residence, it means you have a family and you have a home! [...] We worked for 17 years to create this home then we locked it and fled [...] these matters make you sad (participant 2, man)

Separation from relatives or loved ones who remained back in the country was frequently described as painful. Despite their distance from Syria, migrants remained closely connected to the country and its people. The state of separation from home and loved ones could lead to strong emotions of sadness and joy:

Any news that you hear about the country, whether it's small or big news, it hurts and disturbs the person's mental state, so you find us happy for their happiness and sad for their sadness or their suffering (participant 8 , man)

Other participants called for reunion with loved ones, stating that their mental health state would be resolved if they were able to be reunited with their family members:

If a person could see his children... like for one of our children to come, we probably would not need a psychiatric doctor... you know? Isn't this right? (participant 5, woman)

Struggling for connectedness in a culturally divided world Participants commented on cultural differences and their difficulties in assimilating in the new environment in the 

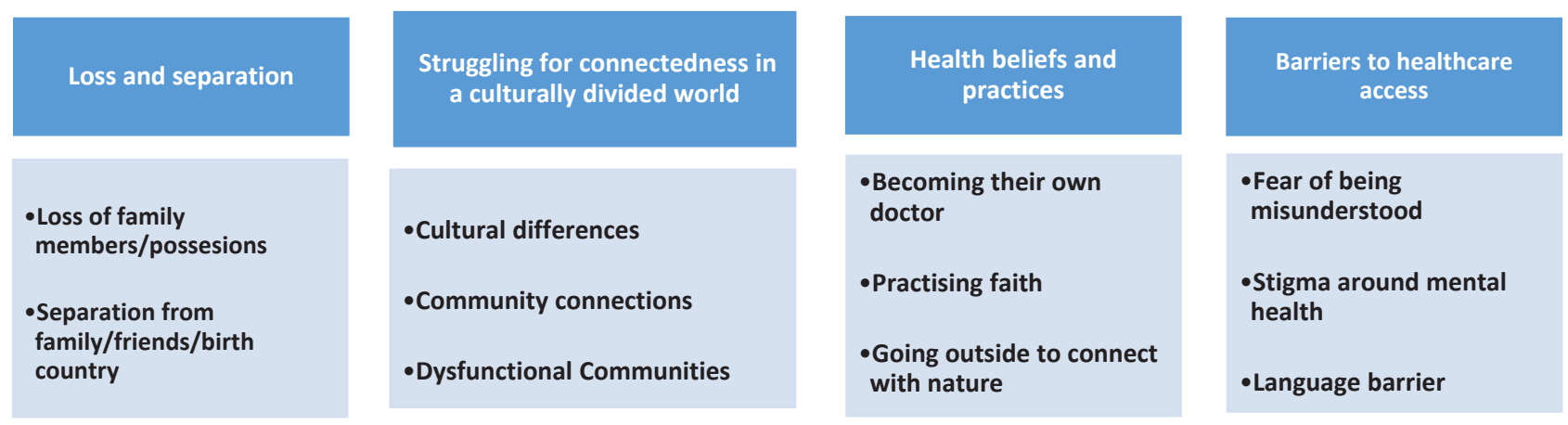

Figure 1: List of key themes and subthemes

Figure 1 List of key themes and subthemes.

new country. There were also comments on feelings of resistance to assimilation on the part of the native population. Despite efforts by Syrians, the gap between the two cultures was considered large and reflected in behaviour and attire:

Integration with the western society or the British community is almost too small... too small... and mostly it's from them, not us... I mean we try, but they as a society have their own costumes and traditions that are completely different than ours (participant 7, man)

One part of this difference lay in divergent priorities in life between the two groups. There was a feeling that Syrians had to live with predicaments that were remote to native British citizens. While Syrians faced diverse social challenges, native people only had to think about employment:

We think of things they do not think about [...] I mean for us as citizens, we can worry about the financial situation, family situation, children, but as a western citizen maybe he would be worried more about his job. I do not see them worrying about the same things (participant 12, man)

Almost all participants talked about how community connection is paramount in preserving the values and cultures of Syrian people as well as a place to share worries and experiences:

We started gathering with the rest of the Syrian families or individuals $[\ldots]$ our encounters begin with one guy on the road or in a certain meeting, certain dinner at a restaurant, sometimes while we are walking we hear his accent so we stop him $[\ldots]$ we are trying as much as possible to gather these people to preserve our customs, traditions, heritage and association with our country (participant 8 , man)

They elaborated on the importance of establishing trusted connections in the community, either with people already in their lives or with new friends. Participants emphasised the significance of these connections in combating disturbances of emotions and stressors:

Being with people with whom you feel loved from, this helps the person a lot to heal or to get out from the situation that he is in (participant 8, man)

However, some participants had a different point of view and remarked that the community feels dysfunctional, as it contains people from different backgrounds with diverse perspectives:

Not all the people who came from Syria have similar characteristics or the same culture, no! ... Today they tell you, but we are a community and we should... ok I get it, but today what is happening — no disrespect-today you are gathering the criminal and the killer, and the doctor and the engineer, and the student and so on. You are putting them in one plate, and you tell us that we are a community, but correct we are a community, but all these ideas, how will you initiate communication between them? (participant 2, man)

Health beliefs and practices

Refugees shared their methods of self-help or what some described as 'becoming their own doctor'. Many shared stories about applying that concept to try to help themselves heal and to forget: 
The person is his own doctor. Whatever happens to you, support or help, if you were not helping yourself from the inside, you won't be able to succeed. You must keep combating in this life, there is no other way (participant 1, woman)

Other expressed their desire to talk to trusted friends or loved ones as a form of therapy. They talked about how getting advice and input from loved ones strengthens the bonds of these relationships and act as a source of releasing pent-up emotions:

I won't let things pile up every day I mean...everyday, everyday, everyday, someone should talk to someone who they feel comfortable with...like your husband or son, daughter, anybody at home...everyday (participant 10, woman)

Appreciation of the health benefits of nature even within urban parts of the UK was conveyed through the interviews and emphasis and encouragement to use nature as a form of healing was seen:

I go to the sea and sit by the sea side, and I express my concerns to the sea, I speak to it. I go to the park, I try to get away from people (participant 8 , man)

Others talked about resorting to faith or faith practices, and the ways these practices can alter their mood and help them:

My mental state is better when I recite the Quran, I continue to do it, it provides comfort for me... Reciting the Quran is a comfort for me... Sometimes I listen to it on the phone, and this is honestly a comfort for me (participant 11, man)

A number of participants strongly emphasised doing something you enjoy as a way to improve mental health. Examples included hobbies, such as reading, writing or a playing a musical instrument.

\section{Barriers to healthcare access}

All refugees showed good knowledge of the healthcare system in general and how to access healthcare facilities. However, there was a general hesitance towards accessing mental healthcare services. Many participants opposed this idea of consulting native British doctors for fear of being misunderstood:

No one would understand what we went through and the situation like you would... who did not witness it won't sympathise, yes, so it's hard for me to go and explain my mental status to a doctor, it's better to explain to God. (participant 11 , man)

Another participant talked about how keeping emotions hidden created a difference between internal and external appearances. This was compared with a new looking phone, but with no battery inside:

I feel that a person is just like the mobile phone where you have to always charge his battery, many times they tell me, wow you look fresh and active today, so I tell them I'm just like the newest version of iPhone, just without a battery (participant 8, man)
Participants talked about how stigma affects their decision to approach mental health services, because of beliefs about mental health informed by growing up in Syria. These participants expressed their concerns of becoming a source of gossip and being labelled as 'crazy':

Back in Syria, people are used to or have the assumption that people who go to psychiatrists have something wrong in their brains, crazy or something [...] so the majority of Syrian people are coy of going to the doctor or are not used to this habit, because we don't have something like that in Syria. (participant 9, man)

On the other hand, half of the participants strongly believed in the notion of breaking taboos regarding mental health. Five of them reported going to a mental health clinic previously in Syria and four described going to a mental health clinic after moving to the UK:

it is necessary to resort to psychiatric medicine... psychiatry is a normal thing and healthy [...] we have a misconception about it like it's for crazy people or so... no, no. On the contrary, it's something healthy and a person must resort to it whenever he feels the need to speak to someone (participant 7 , man)

There was a consensus among all participants that learning the English language was, by far the most difficult barrier faced by the refugees, whether it was in their dayto-day life or in accessing medical healthcare:

I think that's the biggest thing, the language, because medical terminologies you know are very difficult, especially psychological ones [...] the language (participant 7 , man)

Some commented on how the presence of translators can make the challenges of seeking healthcare tougher and that some information could get lost in the translation:

For the translation I think it's not very helpful, sadly... I mean every expression or word I give out has a certain feeling to it, and for a translation it might not give out the proper meaning or it won't come out the intended way, I believe (participant 12, man)

I told them also that the presence of a translator will not make me feel comfortable... sometimes you want to say personal things, or things straight from your heart, so to have a third person as a translator, it will be a bit difficult (participant 10, woman)

Several refugees voiced their stories of struggle in learning the English language despite strenuous efforts, especially as the majority of participants were over 45 years of age. However, they all recognised that it is a necessary component for their integration and life in the UK.

Appreciation of the care received by refugees was a major part of each interview. Participants were grateful for all the efforts given to help them settle in and for the care given to their health: 
What I have seen is that the health care services here are very attentive and they follow up patients [...] The health care system here is very nice. They follow up, since I came here, all the tests I need, the medications I require [...] Their health care system is excellent! (participant 1 , woman)

Participants emphasised that treatment should be based on each case individually. In this way, personalised care was presented as important and something that should be provided to Syrian refugees.

\section{DISCUSSION}

This qulaitative study is the first of its kind to be conducted in the UK on Syrian refugees. Our findings show that refugees face constant challenges as they try to integrate into a new society. Participants described the loss of family members, separation from loved ones in Syria, nostalgia for the homeland as causes of psychological distress and an overwhelming sense of sadness. Struggle for connectedness due to cultural difference and the problematic nature of rapidly formed migrant communities were perceived to hinder effective integration. Participants believed in 'being their own doctor' and turning to faith, ritual and nature for healing and comfort. Taboo and stigma around mental health and language barriers were cited as barriers to accessing mental healthcare services.

Participant narratives in this study reflected existing literature exploring mental health issues in Syrian refugees. ${ }^{24}{ }^{29}$ Previous research on postimmigration factors has highlighted related issues around cultural integration, loss of family and community support, discrimination and adverse political climate. ${ }^{13031}$ Betancourt et al report that many refugees perceive community support as essential to deal with past and present difficulties. ${ }^{32}$ Participants in the present study emphasised the importance of their community not only in coping, unity and support but also as means to preserve traditions and common values. This finding reflects a desire for future generations to remain connected to the homeland. Many participants in Betancourt's study expressed concerns over division in the political, educational and personal stance of other members of their community. Some evidence of discord within the Syrian community was evident in our study. Such differences may lie in kinship groups or friendship networks within communities, which exert a sense of obligation above that of national identity. ${ }^{33}$

Coping mechanisms affect the well-being and mental health status of individuals. Strategies reported among Syrian refugees elsewhere include active and passive ones: cigarette smoking, excessively watching the news, worrying, behavioural withdrawal and 'doing nothing'. ${ }^{34}$ Participants in our study emphasised listening to their own body and trying to cope using non-clinical methods such as using nature, faith practices and hobbies. Using nature for the purpose of improving mental health is a recognised coping strategy, and several studies suggest that it indeed offers benefits. ${ }^{35}$ Barton and Pretty highlighted the importance of 'nature doses' and 'green exercise' in the improvement of mental health and concluded that this approach constituted a means of promoting mental therapy efficiently and easily. ${ }^{36}$ In recent studies of Syrian refugees, faith was reported as a significant coping mechanism. El-Khani et al explored the coping mechanisms used by displaced Syrian refugee mothers in refugee camps and found that parents used adaptation to their circumstances, social support and maintained mental health using faith as a coping strategy. ${ }^{37}$ Similar results were found by Boswall and Al Akash who conducted an ethnographic study on Syrian women in Jordan and found that reading the Quran and practising faith was among the most common coping mechanisms. ${ }^{38}$ This is also shown by Hirschman on his study of immigrants in the USA, which concluded that faith and religious practices provide ethnic communities with refuge from the hostility and discrimination from the host society as well as opportunities for economic mobility and social recognition. ${ }^{39}$

Literature shows that one of the biggest barriers to accessing healthcare in refugee communities is the language barrier, ${ }^{12} 1340-42$ and this was also replicated in our study. Participants commented on the complications created by the presence of an interpreter and raised concerns around poor quality translation as well as confidentiality. One study in the USA on refugees' access to healthcare found that language issues do not only affect access to care and doctor-patient relationship but also restrict the ability to read and understand medical instructions and pharmaceutical prescriptions. ${ }^{43}$ In order to secure the correct diagnosis as well as treatment delivery, interpreters who are familiar with the language and medical terminology need to be consulted. However, the presence of interpreters poses an ethical dilemma of confidentiality breaches and risks altering the relationship between refugee patient and attending physician. ${ }^{44}$ Fatahi et al in their study on Kurdish refugees in Sweden found that participants expressed fear, suspicion and lack of confidence in interpreters. ${ }^{45}$ Bhatia and Wallace found similar results in their study in the UK along with other barriers that refugees face accessing mental healthcare services, for example, stigma and lacking support of a refugee-dedicated agency. ${ }^{46}$ Lack of adequate interpretation complicates patient encounters and elicits fewer empathetic responses from the healthcare provider. In addition to decreasing rapport, it reduces patient satisfaction and increases unintentional medical error. ${ }^{47-49}$ Furthermore, refugees who suffer from PTSD tend to endure greater difficulties in learning the foreign language spoken at the resettlement destination. ${ }^{50}$ Hence, bridging cultural and linguistic differences is integral to improving healthcare access of refugees to eliminate disparities for immigrants and refugees. ${ }^{51} 52$

A key barrier to mental healthcare access by refugees is the stigma. ${ }^{13}$ Our study revealed conflicting views on the value of psychiatry: some participants out rightly rejected mental healthcare treatment for fear of being 
stigmatised, whereas others viewed stigma as an out of date misconception. In Syria and neighbouring countries, it is socially acceptable to express sensitive emotions and emotional suffering. However, labelling those emotions with medical terminologies of distress or as mental health illnesses can lead to shame or embarrassment to the sufferer or their family by being referred to as 'mad' or 'crazy'. ${ }^{53}$ One way to address stigma within refugee populations is through the work of community groups. Palmer and Ward suggested that these groups can assume the role of mediators between refugee service users and the healthcare establishment in order to combat the negative implications of stigma. ${ }^{54}$ In addition, it has been shown that mental health awareness programmes can reduce stigma if provided outside mental health setting, such as in general medical clinics, community centres, schools and women's groups. ${ }^{53}$

Fear of being misunderstood by service care providers was also suggested by refugees as a barrier to accessing mental health facilities, 'no one would understand' being a phrase repeated by participants. Asgary and Segar found similar barriers in their study on refugees and asylum seekers' healthcare access. ${ }^{55}$ A potential solution to this problem would be the involvement of cultural consultants with backgrounds and experience in immigrant and refugee communities in order to provide accurate assessment and improved services to specific communities. ${ }^{56}$ It is crucial for mental health professionals and healthcare service providers to understand that Syrian refugees have a concept of mental illness and psychosocial well-being that is rooted deeply in longstanding social, cultural and religious traditions. ${ }^{57} 58$ Despite the aforementioned barriers, our participants showed an overall good knowledge about the UK National Health Service and how to navigate appointments and access medications. This finding is in contrast with the results of Renner et al in Germany and Saechao et al in the USA who reported a general lack of knowledge of the new healthcare system. ${ }^{1359}$

\section{Strengths and limitations}

A major strength of this study is that it was conducted entirely in the language and dialect of participants. In approaching this study, the researcher adopted the philosophy of Asselin that recommended that an insider researcher should approach a project with 'eyes open' and gather data while assuming no previous knowledge of the phenomenon being studied. ${ }^{28}$ This study, however, also has limitations. One limitation is the small number of participants $(n=12)$. Of the 12 participants interviewed in this study, only 3 were women. Although this might have led to the underrepresentation of the women's experience, this proportion is broadly representative of the refugee population in the UK as the Refugee Council UK suggests that only $26 \%$ of the asylum-seeking applications are made by women. ${ }^{60}$ Recruitment was undertaken during the months of April-June, which coincided with Ramadan (the month of fasting in the Muslim faith), and the Eid celebration (breaking of the fast). This might have influenced participants' decision whether to participate. Nevertheless, the main objective of qualitative research, regardless of sample size, is not to produce a generalisable or statistically significant outcome, but to elicit rich data on which future enquiry can be undertaken. ${ }^{61}$ Another potential limitation is the fact that the study included both newly arrived as well as established refugees (range: 3 months-10 years) and that integration challenges might differ significantly depending on the length of stay in the UK. However, this variation suggests that interventions towards addressing the challenges need to be aimed at an individual level rather than at a group level. The findings of this study may not be generalised to other refugee communities residing in other countries, however, many of the themes identified in this study appear to be common in other refugee studies of different backgrounds. A further limitation is presented in the fact that previous mental health diagnoses among participants were not recorded. This decision was made on grounds of ethics, confidentiality and to promote recruitment. Participant recruitment from this vulnerable group was anticipated to be challenging. Therefore, only basic demographic data were collected in order to promote recruitment and anonymity. This approach inevitably risks participants appearing homogenous. Given the extent of trauma experienced by Syrians and the widespread challenges of integration, it was concluded that all participants would offer valuable narratives. We recognise that for some this could be vicarious in origin but consider it valid because of the close-knit nature of Syrian refugee families/communities and evidence that refugees frequently share experiences/ perceptions. ${ }^{59}$

\section{CONCLUSION}

Findings of this study shed light on the predicament of Syrian refugees in the UK, whose well-being and mental healthcare experiences remain unstudied during integration into the UK society. Participants discussed about mental health distress, especially in terms of separation, loss and a struggle for connectedness. Many factors were found to be helpful or used as coping mechanisms by the refugee community, such as family, nature, faith and pursuing hobbies. However, multiple barriers to accessing healthcare system were identified, in particular, stigma, fear of being misunderstood and language competence. While some of the interviewed refugees had successfully managed to access mental health services, they reported beliefs and challenges that reduced the perceived value of such care. Syrian refugees have become an integral component within British society and it is hoped that this study can serve as a catalyst for further research on this vulnerable group to ensure their proper integration into the UK society.

Twitter Priyamvada Paudyal @paudyal1

Acknowledgements We would like to thank all the participants in this study. Without their cooperation, this study wouldn't have been possible. 
Contributors PP and MJFC designed and supervised the study. MT collected and analysed the data and prepared the initial draft of the paper. PP prepared the final draft with input from MT and MJFC.

Funding The authors have not declared a specific grant for this research from any funding agency in the public, commercial or not-for-profit sectors.

Competing interests None declared.

Patient and public involvement Patients and/or the public were not involved in the design, or conduct, or reporting, or dissemination plans of this research.

Patient consent for publication Not required.

Ethics approval Ethical approval was obtained from the Brighton and Sussex Medical School Research and Ethics Governance Committee (ER/BSMS9DAP/1).

Provenance and peer review Not commissioned; externally peer reviewed.

Data availability statement № data are available. No additional data available.

Open access This is an open access article distributed in accordance with the Creative Commons Attribution Non Commercial (CC BY-NC 4.0) license, which permits others to distribute, remix, adapt, build upon this work non-commercially, and license their derivative works on different terms, provided the original work is properly cited, appropriate credit is given, any changes made indicated, and the use is non-commercial. See: http://creativecommons.org/licenses/by-nc/4.0/.

ORCID iD

Priyamvada Paudyal http://orcid.org/0000-0002-6209-575X

\section{REFERENCES}

1 Abbara A, Coutts A, Fouad FM, et al. Mental health among displaced Syrians: findings from the Syria public health network. $J R$ Soc Med 2016;109:88-90.

2 United Nations High Commissioner for Refugees. Syria emergency: refugees, 2020. Available: https://www.unhcr.org/syria-emergency. html

3 Operational Portal Refugee Situations. Syria regional refugee response. Available: https://data2.unhcr.org/en/situations/syria\#_ga= 2.72926227.1867470304.1601030635-1680491498.1592482273

4 Jankovic J, Bremner S, Bogic M, et al. Trauma and suicidality in war affected communities. Eur Psychiatry 2013;28:514-20.

5 Huijts I, Kleijn WC, van Emmerik AAP, et al. Dealing with manmade trauma: the relationship between coping style, posttraumatic stress, and quality of life in resettled, traumatized refugees in the Netherlands. J Trauma Stress 2012;25:71-8.

6 Teodorescu D-S, Siqveland J, Heir T, et al. Posttraumatic growth, depressive symptoms, posttraumatic stress symptoms, postmigration stressors and quality of life in multi-traumatized psychiatric outpatients with a refugee background in Norway. Health Qual Life Outcomes 2012;10:84.

7 Pascoe EA, Smart Richman L. Perceived discrimination and health: a meta-analytic review. Psychol Bull 2009;135:531-54.

8 Lindencrona F, Ekblad S, Hauff E. Mental health of recently resettled refugees from the middle East in Sweden: the impact of preresettlement trauma, resettlement stress and capacity to handle stress. Soc Psychiatry Psychiatr Epidemiol 2008;43:121-31.

9 Porter M, Haslam N. Predisplacement and postdisplacement factors associated with mental health of refugees and internally displaced persons: a meta-analysis. JAMA 2005;294:602-12.

10 Beiser M, Hou F. Language acquisition, unemployment and depressive disorder among Southeast Asian refugees: a 10-year study. Soc Sci Med 2001;53:1321-34.

11 Tinghög P, Al-Saffar S, Carstensen J, et al. The association of immigrant- and non-immigrant-specific factors with mental ill health among immigrants in Sweden. Int J Soc Psychiatry 2010;56:74-93.

12 Al Ajlan A. Older refugees in Germany: what are the reasons for the difficulties in language-learning? J Refug Stud 2019;17.

13 Renner A, Hoffmann R, Nagl M, et al. Syrian refugees in Germany: perspectives on mental health and coping strategies. J Psychosom Res 2020;129:109906.

14 Burnett $A$, Peel $M$. What brings asylum seekers to the United Kingdom? BMJ 2001;322:485-8.

15 Burnett $A$, Peel $M$. Health needs of asylum seekers and refugees. BMJ 2001;322:544-7.

16 Taylor K. Asylum seekers, refugees, and the politics of access to health care: a UK perspective. Br J Gen Pract 2009;59:765-72.

17 Walker PF, Jaranson J. Refugee and immigrant health care. Med Clin North Am 1999;83:1103-20.
18 Carey-Wood J, Duke KL, Karn V. The settlement of refugees in Britain. HMSO, 1995.

19 GOV.UK. Syrian vulnerable person resettlement programme fact sheet, 2017. Available: https://www.gov.uk/government/ publications/syrian-vulnerable-person-resettlement-programmefact-sheet

20 BBCWorld. Record 400-plus migrants cross channel in one day: BBCWorld, 2020. Available: https://www.bbc.com/news/uk-englandkent-54000755 [Accessed 29 Sep 2020].

21 Georgiadou E, Zbidat A, Schmitt GM, et al. Prevalence of mental distress among Syrian refugees with residence permission in Germany: a registry-based study. Front Psychiatry 2018:9:393.

22 Chung MC, AIQarni N, Al Muhairi S, et al. The relationship between trauma centrality, self-efficacy, posttraumatic stress and psychiatric co-morbidity among Syrian refugees: is gender a moderator? J Psychiatr Res 2017;94:107-15.

23 Alpak G, Unal A, Bulbul F, et al. Post-traumatic stress disorder among Syrian refugees in turkey: a cross-sectional study. Int $J$ Psychiatry Clin Pract 2015;19:45-50.

24 Bogic M, Njoku A, Priebe S. Long-term mental health of warrefugees: a systematic literature review. BMC Int Health Hum Rights 2015;15:29.

25 Rubin HJ, Rubin IS. Qualitative interviewing: the art of hearing data. Sage, 2011.

26 Burnard P. A method of analysing interview transcripts in qualitative research. Nurse Educ Today 1991;11:461-6.

27 Saidin K. Insider researchers: challenges and researchers. Proceedings of the ICECRS 2017;1:25-7.

28 Asselin ME. Insider research: issues to consider when doing qualitative research in your own setting. J Nurses Staff Dev 2003;19:99-103.

29 Ben Farhat J, Blanchet K, Juul Bjertrup P, et al. Syrian refugees in Greece: experience with violence, mental health status, and access to information during the journey and while in Greece. BMC Med 2018;16:40.

30 Kirmayer LJ, Narasiah L, Munoz M, et al. Common mental health problems in immigrants and refugees: general approach in primary care. CMAJ 2011;183:E959-67.

31 Miller KE, Rasmussen A. War exposure, daily stressors, and mental health in conflict and post-conflict settings: bridging the divide between trauma-focused and psychosocial frameworks. Soc Sci Med 2010;70:7-16.

32 Betancourt TS, Frounfelker R, Mishra T, et al. Addressing health disparities in the mental health of refugee children and adolescents through community-based participatory research: a study in 2 communities. Am J Public Health 2015;105 Suppl 3:S475-82.

33 Kelly L. Bosnian refugees in Britain: questioning community. Sociology 2003;37:35-49.

34 United Nations High Commissioner for Refugees. Culture, context and the mental health and psychosocial wellbeing of Syrians: a review for mental health and psychosocial support staff working with Syrians affected by armed conflict. UNHCR, 2020. https:// www.unhcr.org/protection/health/55f6b90f9/culture-context-mentalhealth-psychosocial-wellbeing-syrians-review-mental.html

35 Tillmann S, Tobin D, Avison W, et al. Mental health benefits of interactions with nature in children and teenagers: a systematic review. J Epidemiol Community Health 2018;72:958-66.

36 Barton J, Pretty J. What is the best dose of nature and green exercise for improving mental health? A multi-study analysis. Environ Sci Technol 2010;44:3947-55.

37 El-Khani A, Ulph F, Peters S. Syria: coping mechanisms utilised by displaced refugee parents caring for their children in pre-resettlement contexts. Intervention 2017;15:34-50.

38 Boswall K, Akash RA. Personal perspectives of protracted displacement: an ethnographic insight into the isolation and coping mechanisms of Syrian women and girls living as urban refugees in northern Jordan. Intervention 2015;13:203-15.

39 Hirschman $\mathrm{C}$. The role of religion in the origins and adaptation of immigrant groups in the United States. Int Migr Rev 2004;38:1206-33.

40 Sheikh-Mohammed M, Macintyre CR, Wood NJ, et al. Barriers to access to health care for newly resettled sub-Saharan refugees in Australia. Med J Aust 2006;185:594-7.

41 Lipson JG, Omidian PA. Health issues of Afghan refugees in California. West J Med 1992;157:271-5.

42 Segal UA, Mayadas NS. Assessment of issues facing immigrant and refugee families. Child Welfare 2005;84:563-83.

43 Morris MD, Popper ST, Rodwell TC. Healthcare barriers of refugees post-resettlement. J Community Health 2009;34:529-38.

44 Jefee-Bahloul H, Moustafa MK, Shebl FM, et al. Pilot assessment and survey of Syrian refugees' psychological stress and openness 
to referral for telepsychiatry (PASSPORT study). Telemed J E Health 2014;20:977-9.

45 Fatahi N, Nordholm L, Mattsson B, et al. Experiences of kurdish war-wounded refugees in communication with Swedish authorities through interpreter. Patient Educ Couns 2010;78:160-5.

46 Bhatia R, Wallace P. Experiences of refugees and asylum seekers in general practice: a qualitative study. BMC Fam Pract 2007;8:48.

47 Wissink L, Jones-Webb R, DuBois D, et al. Improving health care provision to somali refugee women. Minn Med 2005;88:36-40.

48 Ngo-Metzger Q, Sorkin DH, Phillips RS, et al. Providing highquality care for limited english proficient patients: the importance of language concordance and interpreter use. J Gen Intern Med 2007;22 Suppl 2:324-30.

49 MacFarlane A, Glynn LG, Mosinkie PI, et al. Responses to language barriers in consultations with refugees and asylum seekers: a telephone survey of Irish general practitioners. BMC Fam Pract 2008;9:68.

50 Söndergaard HP, Theorell T. Language acquisition in relation to cumulative posttraumatic stress disorder symptom load over time in a sample of re-settled refugees. Psychother Psychosom 2004;73:320-3.

51 Williams DR, Jackson PB. Social sources of racial disparities in health. Health Aff 2005;24:325-34.

52 Barr DA, Wanat SF. Listening to patients: cultural and linguistic barriers to health care access. Fam Med 2005;37:199-204.
53 Hassan G, Ventevogel P, Jefee-Bahloul H, et al. Mental health and psychosocial wellbeing of Syrians affected by armed conflict. Epidemiol Psychiatr Sci 2016;25:129-41.

54 Palmer D, Ward K. 'Lost': listening to the voices and mental health needs of forced migrants in London. Med Confl Surviv 2007;23:198-212.

55 Asgary R, Segar N. Barriers to health care access among refugee asylum seekers. J Health Care Poor Underserved 2011;22:506-22.

56 Kirmayer LJ, Groleau D, Guzder J, et al. Cultural consultation: a model of mental health service for multicultural societies. Can $J$ Psychiatry 2003;48:145-53.

57 Al Adawi S. Caring for Arab patients: a biopsychosocial approach. Sultan Qaboos Univ Med J 2008;8:233-4.

58 Kirmayer LJ. Rethinking cultural competence. Transcult Psychiatry 2012;49:149-64.

59 Saechao F, Sharrock S, Reicherter D, et al. Stressors and barriers to using mental health services among diverse groups of firstgeneration immigrants to the United States. Community Ment Health J 2012;48:98-106.

60 The Migration Observatory. The migration observatory informs debates on international migration and public policy. Asylum and refugee resettlement in the UK - Migration Observatory. MigObs, 2021. https://migrationobservatory.ox.ac.uk/resources/briefings/ migration-to-the-uk-asylum/

61 Anastas JW. Research design for social work and the human services. Columbia University Press, 1999. 